\title{
Continuous Supplementation of Milk Fat Globule Membrane with Habitual Exercise from a Young Age Improves Motor Coordination and Skeletal Muscle Function in Aged Mice
}

\author{
Michiko Yano, Satoshi HARAmizu, Noriyasu OTA*, \\ Yoshihiko MiNeGiSHI and Akira SHIMOTOYODOME \\ Biological Science Research, Health Science, Kao Corporation Tochigi Research Center, \\ 2606 Akabane, Ichikai-machi, Haga-gun, Tochigi 321-3497, Japan
}

(Received January 9, 2019)

\begin{abstract}
Summary Since the decline of physical performance gradually progresses with aging, continuous exercise with nutritional supplementation from a young age is a feasible and effective way to maintain a comfortable life until late old age. We examined the effects of continuous milk fat globule membrane (MFGM) supplementation combined with voluntary running exercise (VR) for prevention of aging-associated declines in physical performance in naturally aging mice. The MFGM with VR group showed a significantly attenuated age-related decline in motor coordination and suppression of the loss of muscle mass and strength. Compared with the control group, the MFGM with VR group showed significantly higher mRNA and protein expression for docking protein 7 , which maintains neuromuscular junction (NMJ) integrity, in the quadriceps muscles. These results suggest that dietary MFGM and VR attenuate natural aging-related decline in motor coordination and muscle function by regulating NMJ integrity.
\end{abstract}

Key Words aging, docking protein 7, neuromuscular junction, nutrition, physical performance

Physical performance undergoes progressive deterioration during aging, which causes a significant decline in the quality of life and increases risks of falling, disability, and death (1). Given that populations worldwide are rapidly aging, there is a growing need to find ways to attenuate age-related physical disability. Physiological impairments, such as age-related loss of skeletal muscle mass and function, are likely to cause a decline in physical performance (2), including motor coordination, which is highly related to the risk of falling $(3,4)$. Accumulating evidence from animal and human studies suggests that skeletal muscle aging is closely associated with degeneration of the morphological and functional integrity of the neuromuscular junction (NMJ) $(5,6)$. NMJ degradation leads to reduced motor unit activity. A motor unit consists of an individual motor neuron and the muscle fibers that it innervates $(7,8)$. The loss of functional integrity in motor units is closely related to decreased muscle function and motor coordination $(9$, 10). Hence, maintaining NMJ integrity and motor unit function may be an effective means of combating agerelated declines in physical performance.

Aging-associated declines in motor coordination and muscle function can be improved by lifestyle interventions such as exercise and nutritional supplementation, even in older adults $(11,12)$. Resistance training or moderate exercise can improve muscle mass and strength and decrease frailty (13-15). In young

*To whom correspondence should be addressed.

E-mail: ota.noriyasu@kao.com adults, whole milk consumption after resistance training improves body composition, protein synthesis, and muscle mass (16-18). However, when such training is combined with daily consumption of low-fat milk, these effects are not enhanced (19). Thus, the beneficial effects of dietary milk supplementation on skeletal muscle mass and function remain controversial.

Milk contains approximately 3-5\% fat in a stabilized emulsion of tiny globules. A membrane surrounds the triglyceride core of each fat globule and is referred to as the milk fat globule membrane (MFGM), which contains unique polar lipids and membrane-specific proteins (20). We previously reported that dietary MFGM combined with moderate exercise improved physical performance in older (21-23) and healthy middle-aged adults (24). Our previous animal research demonstrated that dietary MFGM supplementation combined with voluntary exercise suppressed age-related deficits in muscle mass and function in senescence-accelerated mice (SAM). These changes were associated with increased expression of genes involved in maintaining the integrity of the NMJ (25). The SAM mice have been developed through selective inbreeding of the AKR/J strain of mice based on the data of the grading score of senescence, life span, and pathologic phenotypes (26). SAM mice consist of two strains, the senescence-accelerated prone mice (SAMP) and senescence-accelerated resistant mice (SAMR). Compared with SAMR strains, the control strains that ages normally, the SAMP strains show a rapid progression of senescence, higher oxidative stress, and a shorter median life span. Although SAM mice are a widely used 
animal model for understanding age-related conditions, the clinical relevance of this model has not been sufficiently proven (27). It is, therefore, meaningful to verify the above results using naturally aging mice, which can increase the validity of evidence. In addition, motor coordination has not been evaluated using SAM mice (25).

Motor coordination begins to decline after $50 \mathrm{y}$ of age, with more drastic declines occurring over the age of $70 \mathrm{y}$ in humans (28). Accordingly, preventing the accelerated decline of motor coordination in the later phases of aging may greatly contribute to reducing the risk of falls in older adults. Justice et al. (28) also demonstrated that the rate at which motor coordination declines among mice aged 20-26 mo was equivalent to that among humans aged 70-89 y. Our recent study demonstrated that MFGM intake combined with voluntary exercise ameliorated age-related deterioration in motor coordination and muscle strength of naturally aging mice during the early phase of aging (at 14 mo of age) (29). However, the effect of MFGM intake plus exercise on much older mice has not been clarified. Therefore, we tested the hypothesis that continuous MFGM supplementation combined with voluntary running (VR) from a young age prevents aging-associated declines in motor coordination and skeletal muscle function of naturally aging mice in the later phase of aging (at 20-21 mo of age).

\section{MATERIALS AND METHODS}

Materials. MFGM was prepared from buttermilk by filtration, centrifugation, and drying. The composition of MFGM was analysed at the Japan Food Research Laboratories foundation (Tokyo, Japan). The MFGM contained $46.9 \%$ protein, $35.7 \%$ fat (16.6\% phospholipids, comprising $4.71 \%$ phosphatidylcholine, 5.2\% phosphatidylethanolamine, $1.32 \%$ phosphatidylinositol, $1.74 \%$ phosphatidylserine, and $3.62 \%$ sphingomyelin), $10.6 \%$ carbohydrates, $3.8 \%$ ash, and 3.0\% moisture, among other components. The fatty acid composition was described previously (30).

Animals and experimental design. Two-month-old male C57BL/6J mice were purchased from Charles River Japan, Inc. (Yokohama, Japan) and maintained in an environment in which temperature $\left(23 \pm 2^{\circ} \mathrm{C}\right)$, humidity (55 $\pm 10 \%$ ), and lighting (lights on from 07:00 to 19:00) were controlled. The mice were fed laboratory chow (CE2; CLEA Japan, Inc., Tokyo, Japan) and had free access to drinking water for $1 \mathrm{wk}$ to acclimate to their new environment. After acclimation, the mice were weighed, and those $6.5 \%$ heavier or $10.5 \%$ lighter than average were removed to minimize individual differences in body weight. In total, 32 mice with similar body weights were selected and randomly allocated to the following groups ( $n=8$ per group): the Cont_Sed group, which was fed a control diet containing $10 \%$ fat (w/w), 20\% casein, $55.5 \%$ potato starch, $8.1 \%$ cellulose, $4 \%$ minerals, $2.2 \%$ vitamins, and $0.2 \%$ methionine, and housed in a standard plastic cage (TP-106; $175 \times 245 \times 125 \mathrm{~mm}$; Toyoriko, Co., Tokyo, Japan); the MFGM_Sed group, which was fed the MFGM diet, consisting of the control diet supplemented with 1\% MFGM, and housed in a standard cage; the Cont_VR group, which was fed the control diet and housed in a similar cage equipped with a running wheel for VR (SW-15s; Melquest, Co., Toyama, Japan); and the MFGM_VR group, which was fed the MFGM diet and also housed in a running-wheel cage. For 19 mo (from 2 to 21 mo of age), the mice were allowed ad libitum access to water and the control or MFGM diet, both of which were in powder form. Another group, consisting of 2-mo-old mice (the Young_Sed group, $n=8$ ), was used for observing the natural influence of aging and was allowed ad libitum access to water and the control diet for 2 mo (up to 4 mo of age). Each cage had a nest box (Shepherd Specialty Papers, Inc., Watertown, TN, USA) for environmental enrichment. Dietary intake was measured throughout the experimental period by subtracting the weight of the remaining food from the weight of the food provided on the previous feeding day. At 20 mo of age, the VR volume of each mouse in the Cont_VR and MFGM_VR groups were recorded for $3 \mathrm{~d}$, and the average VR counts (counts/d) were calculated. All animal experiments were conducted in the Experimental Animal Facility of the Kao Corporation Research and Development Department. All experiments were in strict accordance with the guidelines of the Kao Corporation Animal Care Committee, which approved this study (approval number: N2012-0013A).

Rotarod test. Motor coordination (31) of the mice was evaluated at 3 and 20 mo of age (the latter being 18 mo after the beginning of the experiment) using a rotarod (MK-600; Muromachi Kikai, Co., Tokyo, Japan). After a 1-wk period during which the mice were allowed to acclimate to the rotarod (set to $20 \mathrm{rpm}$ ), we measured the total amount of time (min) spent on the apparatus before falling off 10 or 15 times. The rotation speed was initially kept at $20 \mathrm{rpm}$ for $30 \mathrm{~min}$, before being incrementally increased to $24 \mathrm{rpm}$ and maintained at this level for $90 \mathrm{~min}$. Subsequently, it was increased to, and maintained at, $28 \mathrm{rpm}$ until the mouse fell off. We included an interval of 30-60 s between falling off and the next trial to minimize stress.

Tissue collection. The mice were weighed at 4 (in the case of the Young_Sed group) or 21 mo of age (for all other groups). Under $2 \%$ isoflurane inhalation-induced anesthesia (Abbott Japan, Co., Tokyo, Japan), non-fasted mice were euthanized by exsanguination via the postcaval vein. The quadriceps, gastrocnemius, plantaris, and soleus muscles; epididymal and perirenal white adipose tissues; and liver were resected and weighed. The tissue samples were stored at $-80^{\circ} \mathrm{C}$ until analysis.

Muscle contractile force measurement. Muscle contractile force was measured as described previously (32). Immediately after its isolation, the soleus muscle of the right leg was anchored horizontally between two hooks, one fixed and the other attached to an isometric force transducer (World Precision Instruments, Inc., Sarasota, FL, USA), and immersed in Krebs solution. The solution was continuously bubbled with 95\% $\mathrm{O}_{2} / 5 \% \quad \mathrm{CO}_{2}$ at $37^{\circ} \mathrm{C}$. The muscle was electrically stimulated with a 
Table 1. Body and tissue weights and dietary intake of mice in the five experimental groups.

\begin{tabular}{lccccc}
\hline & Young_Sed & Cont_Sed & MFGM_Sed & Cont_VR & MFGM_VR \\
\hline Initial body weight, g & not assigned & $24.9 \pm 0.3$ & $24.9 \pm 0.4$ & $24.9 \pm 0.3$ & $24.9 \pm 0.3$ \\
Final body weight, g & $35.0 \pm 1.0$ & $43.1 \pm 4.9$ & $39.9 \pm 4.1$ & $37.5 \pm 3.6$ & $34.7 \pm 1.2$ \\
Dietary intake, g & $3.7 \pm 0.2$ & $3.6 \pm 0.2$ & $3.7 \pm 0.1$ & $3.3 \pm 0.1$ & $3.5 \pm 0.1$ \\
Quadriceps, mg & $399.3 \pm 8.7^{*}$ & $299.2 \pm 24.5$ & $305.0 \pm 16.7$ & $339.6 \pm 15.3$ & $377.9 \pm 3.4^{*}$ \\
Gastrocnemius, mg & $279.3 \pm 2.9$ & $243.3 \pm 16.3$ & $241.8 \pm 15.3$ & $259.9 \pm 9.1$ & $276.0 \pm 7.1$ \\
Plantaris, mg & $39.1 \pm 0.6$ & $29.9 \pm 1.6$ & $30.5 \pm 2.1$ & $34.0 \pm 1.3$ & $37.8 \pm 1.3$ \\
Soleus, mg & $20.1 \pm 0.7$ & $19.3 \pm 0.9$ & $19.3 \pm 1.2$ & $22.6 \pm 1.0$ & $22.2 \pm 1.5$ \\
Total muscles, mg & $738.0 \pm 8.6^{*}$ & $594.5 \pm 40.0$ & $597.8 \pm 34.1$ & $657.9 \pm 24.8$ & $714.5 \pm 10.4^{*}$ \\
Epididymal fat, g & $1.41 \pm 0.15$ & $1.47 \pm 0.55$ & $1.25 \pm 0.41$ & $1.15 \pm 0.33$ & $0.60 \pm 0.17$ \\
Perirenal fat, g & $0.66 \pm 0.08$ & $1.03 \pm 0.39$ & $0.95 \pm 0.30$ & $0.73 \pm 0.25$ & $0.31 \pm 0.04$ \\
Liver, g & $1.26 \pm 0.09$ & $1.74 \pm 0.17$ & $1.81 \pm 0.22$ & $1.55 \pm 0.24$ & $1.41 \pm 0.06$ \\
\hline
\end{tabular}

Values are presented as means \pm SE $(n=5-8) .{ }^{*} p<0.05$ vs. Cont_Sed group according to Dunnett’s test. Young_Sed, young mice without voluntary running (VR); Cont_Sed, aged mice fed the control diet without VR; MFGM_Sed, aged mice fed the MFGM diet without VR; Cont_VR, aged mice fed the control diet with VR; MFGM_VR, aged mice fed the MFGM diet with VR.

stimulus isolation unit (SEN-3301; Nihon Kohden, Inc., Tokyo, Japan). Twitch force was measured with a single pulse, whereas tetanic responses were induced with a 330 -ms series of 0.2 -ms pulses at $140 \mathrm{~Hz}$ delivered once every $2 \mathrm{~s}$ and digitally recorded for 2 min using a bridge amplifier and data acquisition system (Quad-16I; World Precision Instruments, Inc.). The acquired data were analyzed using Data-Trax software (World Precision Instruments, Inc.).

Quantitative reverse-transcription polymerase chain reaction ( $q R T-P C R)$. Total RNA was extracted using a RNeasy Fibrous Tissue Mini Kit (Qiagen, Inc., Hilden, Germany) from the quadriceps and soleus muscles. qRT-PCR was conducted with SYBR Green PCR Master Mix (Applied Biosystems, Inc., Foster City, CA, USA) on an ABI ViiA 7 Real-Time PCR System (Life Technologies Japan, Tokyo, Japan), as described previously $(25,32)$. All expression data were normalized to those for the gene encoding acidic ribosomal protein P0 (Rplp0/36b4). The sequences of the primers used were as follows: docking protein 7 (Dok-7) forward, TGAGCTTCCTGTTTGACTGCA; Dok-7 reverse, GCAACACGCTCTTCTGAGGC; ciliary neurotrophic factor (Cntf) forward, GGTTGACCCACGTATGAACA; Cntf reverse, CCACCAATACAAACAAAATCATGTA; muscle-specific receptor tyrosine kinase (MUSK) forward, CCACACTGCGTGGAATGAGC; MuSK reverse, CTCTGCAAATGGGCATGGG; Rplp0/36b4 forward, GACATCACAGAGCAGGCCCT; Rplp0/36b4 reverse, TCTCCACAGACAATGCCAGG.

Western blot analysis. The quadriceps muscles of mice in all groups were analyzed for protein expression by western blot analysis, as described previously (25). Goat polyclonal anti-Dok-7 (1 : 1,000 dilution; Santa Cruz Biotechnology, Inc., Dallas, TX, USA), rabbit polyclonal anti-Cntf ( 1 : 1,000 dilution; Abcam, Cambridge, MA) and rabbit monoclonal anti-glyceraldehyde 3-phosphate dehydrogenase (GAPDH, 1: 5,000 dilution; Cell Signaling Technology, Inc., Beverly, MA, USA) primary antibodies were used to recognize each protein. For

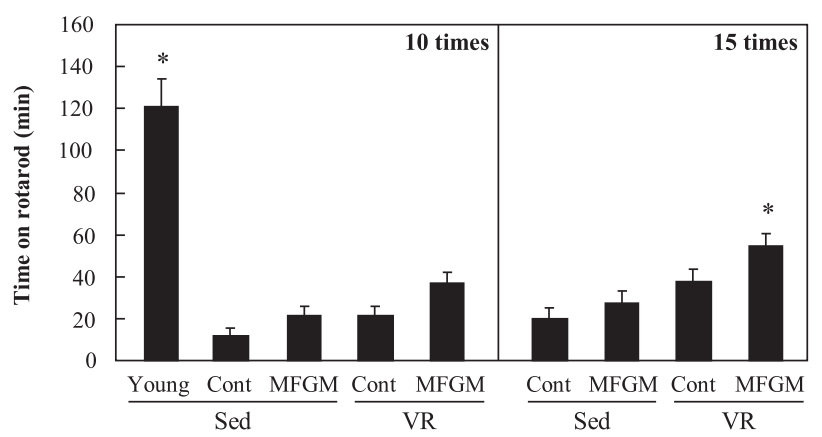

Fig. 1. Effects of milk fat globule membrane (MFGM) on motor coordination. We measured the total time (min) spent on a rotarod until 10 or 15 falls. Values are presented as means \pm standard errors $(n=5-8) .{ }^{*} p<0.05$ vs. Cont_Sed group according to Dunnett's test. Young_Sed, young mice without voluntary running (VR); Cont_Sed, aged mice fed the control diet without VR; MFGM_Sed, aged mice fed the MFGM diet without VR; Cont_VR, aged mice fed the control diet with VR; MFGM_VR, aged mice fed the MFGM diet with VR.

detection of Dok-7 antibody binding, horseradish peroxidase-labelled anti-goat immunoglobulin antibodies (1 : 5,000 dilution; R\&D Systems, Inc., Minneapolis, MN, USA) were used. For detection of Cntf and GAPDH, anti-rabbit immunoglobulin antibodies (1 : 5,000 dilution; Cell Signaling Technology, Inc.) were used. Dok-7 and Cntf protein expression data were normalized to levels of the GAPDH protein.

Statistical analysis. All values are presented as means \pm standard errors (SE). To compare the means of more than two groups, we used an analysis of variance, followed by Dunnett's test (StatView for Windows Version 5.0; SAS Institute, Co., Cary, NC, USA). Correlations were analyzed using Pearson's correlation coefficient. A $p$-value $<0.05$ was considered statistically significant. 
Table 2. Contractile force produced by the soleus muscle.

\begin{tabular}{lccccc}
\hline & Young_Sed & Cont_Sed & MFGM_Sed & Cont_VR & MFGM_VR \\
\hline Maximum force, g & $22.06 \pm 0.85^{*}$ & $15.17 \pm 1.16$ & $18.41 \pm 1.76$ & $18.88 \pm 1.45$ & $21.61 \pm 1.00^{*}$ \\
Force after 10 s, g & $18.75 \pm 0.88^{*}$ & $12.26 \pm 1.42$ & $15.90 \pm 1.99$ & $15.39 \pm 1.55$ & $20.00 \pm 1.35^{*}$ \\
Force after 20 s, g & $16.49 \pm 0.87^{*}$ & $11.14 \pm 1.55$ & $14.47 \pm 1.83$ & $13.31 \pm 1.21$ & $18.16 \pm 1.25^{*}$ \\
\hline
\end{tabular}

Values are presented as means \pm SE $(n=5-8) .{ }^{*} p<0.05$ vs. Cont_Sed group according to Dunnett's test. Young_Sed, young mice without voluntary running (VR); Cont_Sed, aged mice fed the control diet without VR; MFGM_Sed, aged mice fed the MFGM diet without VR; Cont_VR, aged mice fed the control diet with VR; MFGM_VR, aged mice fed the MFGM diet with VR.
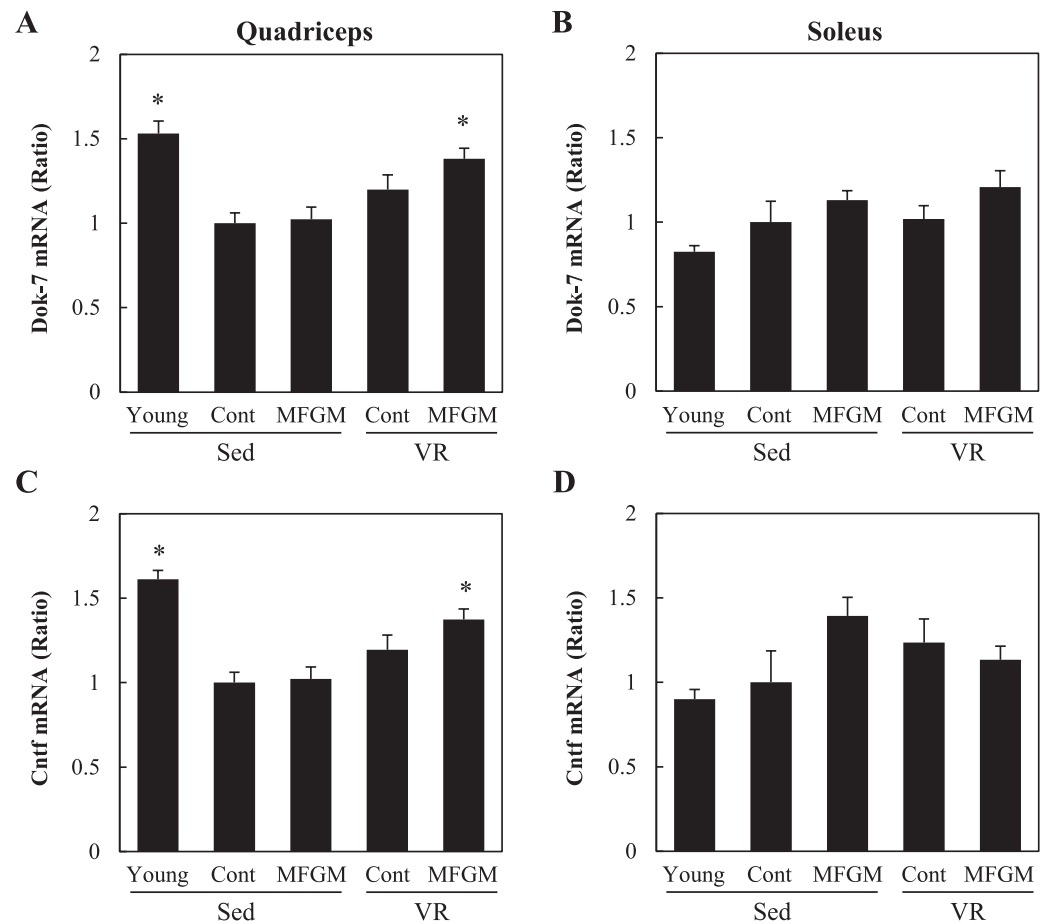

D
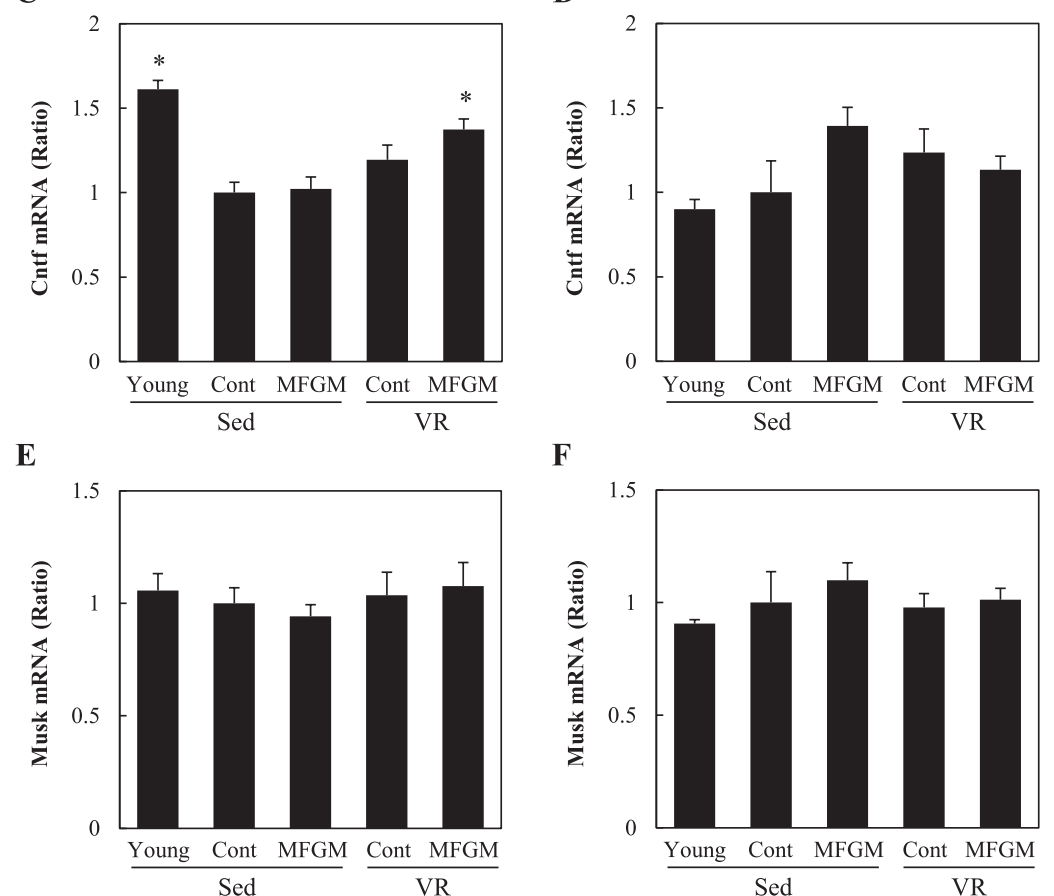

$\mathbf{F}$

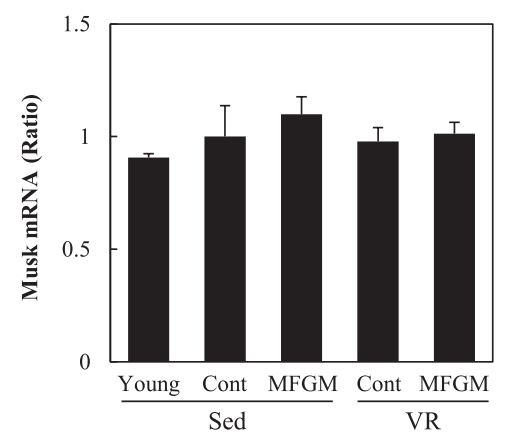

Fig. 2. Effects of milk fat globule membrane (MFGM) on mRNA expression of neuromuscular junction (NMJ)-related genes in skeletal muscles. We quantified docking protein 7 (Dok-7, A and B), ciliary neurotrophic factor (Cntf, C and D), and muscle-specific receptor tyrosine kinase (MuSK, E and F) mRNA expression in quadriceps (A, C, E) and soleus (B, D, F) muscles. Values are presented as means \pm standard errors $(n=5-8) .{ }^{*} p<0.05$ vs. Cont_Sed group according to Dunnett's test. Values are expressed as ratios to the Cont_Sed group, which was set as 1.0. Young_Sed, young mice without voluntary running (VR); Cont_Sed, aged mice fed the control diet without VR; MFGM_Sed, aged mice fed the MFGM diet without VR; Cont_VR, aged mice fed the control diet with VR; MFGM_VR, aged mice fed the MFGM diet with VR.

\section{RESULTS}

Effect of MFGM with VR on mouse body and tissue weights during natural aging

Of the eight mice in each group, five in Cont_Sed, seven in MFGM_Sed, seven in Cont_VR, and six in MFGM_VR survived. Quadriceps and total muscle weights were significantly higher in the Young_Sed and MFGM_VR groups than in the Cont_Sed group $(p<0.05$; Table 1). Body weight, dietary intake, and other tissue weights did not differ among the groups (Table 1). There were no statistically significant differences in the average VR 
A

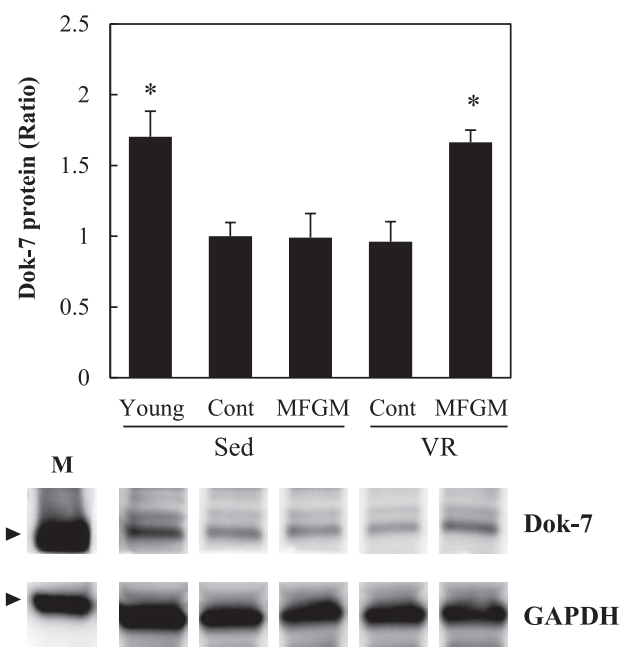

B

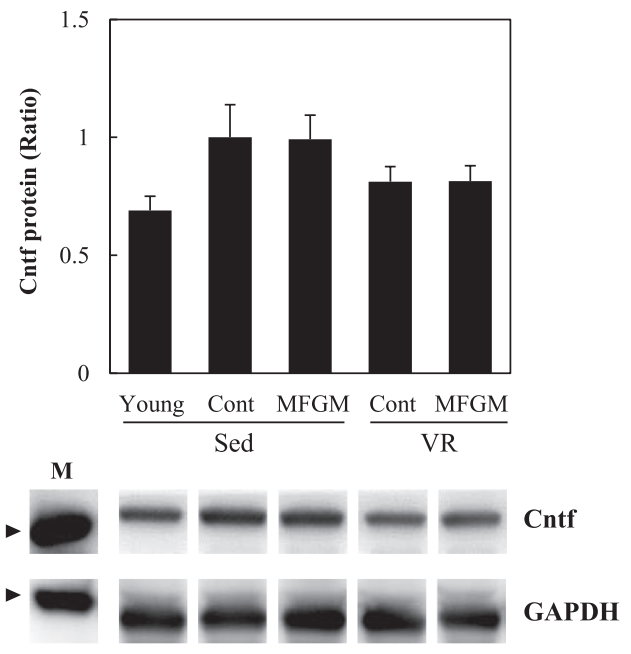

Fig. 3. Effects of milk fat globule membrane (MFGM) on protein expression of neuromuscular junction (NMJ)related molecules in skeletal muscles. We quantified docking protein 7 (Dok-7, A) and ciliary neurotrophic factor (Cntf, B) protein expression in quadriceps muscles. Values are presented as means \pm standard errors $(n=5-8) .{ }^{*} p<0.05$ vs. Cont_Sed group according to Dunnett's test. Values are expressed as ratios to the Cont_Sed group, which was set as 1.0. Young_Sed, young mice without voluntary running (VR); Cont_ Sed, aged mice fed the control diet without VR; MFGM Sed, aged mice fed the MFGM diet without VR; Cont_ VR, aged mice fed the control diet with VR; MFGM_VR, aged mice fed the MFGM diet with VR; M, molecular weight marker; GAPDH, glyceraldehyde 3-phosphate dehydrogenase.

counts exhibited by mice at 20 mo of age in the Cont VR and MFGM_VR groups (Cont_VR: 2,278 2386 , and MFGM_VR: 1,850 \pm 304 counts/d, respectively).

Effect of MFGM with VR on motor coordination and tetanic contractile force of soleus muscle

Concerning motor coordination, the total time spent by mice on the rotarod until falling off ten times was significantly $(p<0.05)$ longer in the Young_Sed than in the Cont_Sed group $(p<0.05$; Fig. 1). Young_Sed mice were able to stay on the rotarod ten times longer than Cont_Sed mice. Young_Sed mice were excluded from further analysis in these tests because they did not fall off the rotarod 15 times, even after $180 \mathrm{~min}$. The total time spent on the rotarod until falling off 15 times was significantly longer in the MFGM_VR than the Cont_Sed group ( $p<0.05$; Fig. 1). The tetanic contractile force of the soleus muscle was significantly greater in the Young_Sed and MFGM_VR groups than in the Cont_ Sed group ( $p<0.05$; Table 2).

Effects of MFGM with VR on NMJ-related gene and protein expression in skeletal muscle

Dok-7 mRNA (Fig. 2A) and protein (Fig. 3A) levels in quadriceps muscles were significantly higher in the Young_Sed and MFGM_VR groups than in the Cont_Sed group $(p<0.05)$. Cntf mRNA level in quadriceps muscles (Fig. 2C) were higher in the Young_Sed and MFGM_VR groups than in the Cont_Sed group $(p<0.05)$, on the other hand, there were no significant changes in protein levels of the quadriceps muscles (Fig. 3B). There were no between-group differences in mRNA levels of MuSK in the quadriceps muscles (Fig. 2E). No significant changes were observed in mRNA levels of the soleus muscles (Fig. 2B, D, F).

Correlation between motor coordination and conditions of skeletal muscle

The total time spent on the rotarod until falling off 15 times showed a significant positive correlation with Dok-7 mRNA (Fig. 4A) and protein (Fig. 4C) levels in the quadriceps muscles $(p<0.05)$, but not with Dok-7 mRNA level in the soleus muscles (Fig. 4B). Neither the weight of the quadriceps muscle (Fig. 4E) nor the contractile force of the soleus muscle (Fig. 4D) correlated with total time spent on the rotarod. The weight of the other muscles (gastrocnemius, plantaris, and total muscles) did not correlate with total time spent on the rotarod (data not shown).

\section{DISCUSSION}

The present study produced three major findings. First, we demonstrated that continuous MFGM supplementation with VR prevented aging-related decline in motor coordination of naturally aging mice in the later phase of aging. Motor coordination, assessed as time spent on the rotarod, dramatically deteriorated with natural aging in mice. Similar results have been reported in humans (33). Structural and functional deterioration of the skeletal muscles during aging impairs the functional output of motor units, which results in compromised motor coordination (10). Recently, we demonstrated that MFGM nutritional supplementation with habitual exercise increased motor unit recruitment and activity in humans and may thereby improve agility (22, 34). Our current results support the involvement of improved motor unit function in increased physical performance, including motor coordination, after MFGM consumption with habitual exercise.

Second, dietary MFGM supplementation combined with VR attenuated aging-associated loss of muscle mass and strength in naturally aging mice, consistent 
A

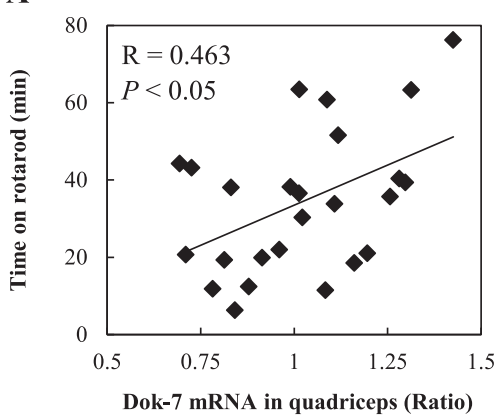

C

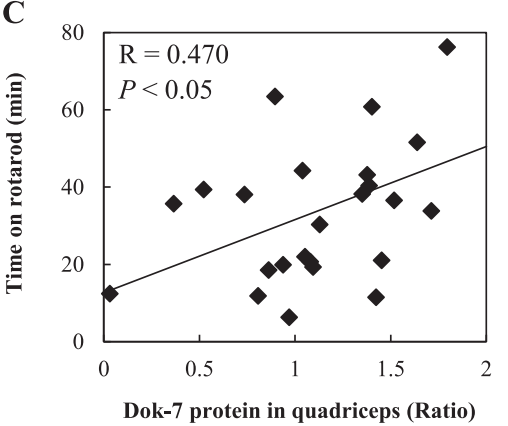

E

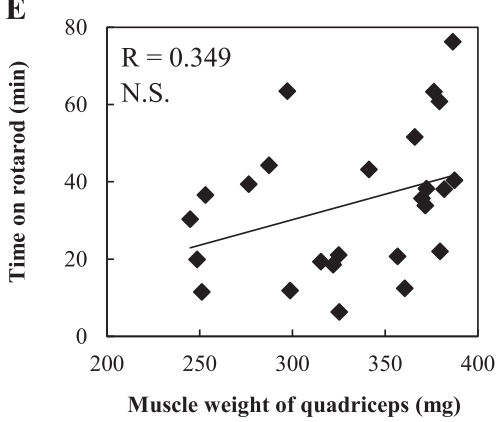

B

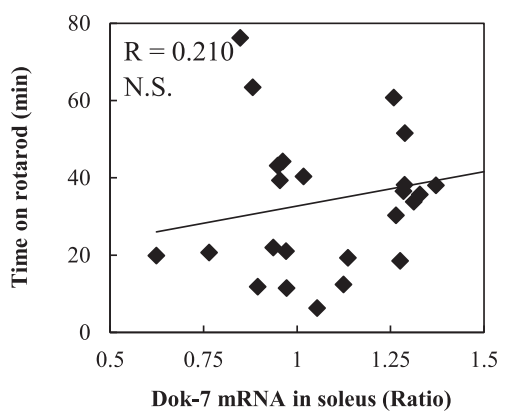

D

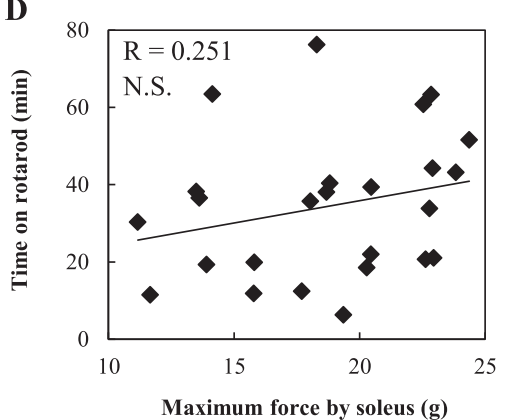

Fig. 4. Correlation between motor coordination and conditions of skeletal muscle. We analysed the correlation between the total time spent on the rotarod until falling off 15 times and Dok-7 mRNA (A and B) and protein (C) expression in quadriceps (A and C) and soleus (B) muscles. We also analysed the correlation between the total time spent on the rotarod and the contractile force of soleus muscle (D) and the weight of quadriceps muscle (E). Correlations were analysed using Pearson's correlation coefficient, and $* p<0.05$ was considered statistically significant $(n=25)$. R, correlation coefficient.

with our previous findings in SAM mice (25). MFGM intake or VR alone did not significantly improve muscle mass and strength, which is in agreement with previous studies $(29,35-37)$. These results suggest that improvements in muscle characteristics that are associated with MFGM ingestion and VR depend on the synergism between these factors. In the present study, neither the muscle weight nor muscle contractile forces were associated with the time spent on the rotarod; therefore, the improvement in motor coordination caused by dietary MFGM with VR may be related to other factors.

Finally, we found that Dok-7, which is associated with maintaining the integrity of the NMJ (38-40), was upregulated in the quadriceps muscle tissue of naturally aging mice that received MFGM supplemented with VR, consistent with the results of our previous study that used SAM mice (25). Moreover, a significant correlation between motor coordination and Dok-7 expression levels in quadriceps muscles was observed in the present study. Dok-7 and MuSK are colocalized with acetylcholine receptors (AChRs) at the postsynaptic area in skeletal muscle, and clustering formation of AChRs is a crucial step in NMJ formation (41). MuSK activated by Dok-7 and agrin can induce AChR clustering; therefore, the Dok-7/MuSK pathway plays an essential role in maintaining NMJ integrity. Arimura et al. (38) have shown that Dok-7 overexpression dramatically improves NMJ morphology, and consequently, muscle function and motor coordination in lamin A/C knockout mice, a mouse model of neuromuscular disorders. As NMJ integrity is associated with motor unit function (8), MFGM intake with VR may increase Dok-7 production in skeletal muscles as in case of the quadriceps muscle, thereby improving NMJ integrity and motor unit function, resulting in attenuation of age-related declines in motor coordination and skeletal muscle function. Although we also assessed expression levels of other molecules involved in maintaining NMJ integrity, including MuSK and Cntf (42), we did not find the molecule which showed significant changes in both mRNA and protein levels. These results suggest that Dok-7 is a specific target of MFGM intake with VR. 
In the present study, increase of muscle weight and Dok-7 expression level caused by MFGM supplementation with VR were observed in the quadriceps muscle, but not in soleus muscle. Skeletal muscles consist of different fiber types: slow-twitch fiber and fast-twitch fiber. Soleus muscle consists of predominantly slow-twitch fibers; quadriceps muscle displays a mixed proportion of muscle fibers and contains more fast-twitch fibers than soleus muscle. NMJ abnormalities are more frequently observed in extensor digitorum longus muscles, which composed of predominantly fast-twitch fibers, than soleus muscle (43). Preferential loss of fast-twitch muscle fibers occurs during aging and causes age-associated muscle atrophy (44), consistent with our result that decrease of muscle weight during aging was observed only in the quadriceps muscle. These findings suggest that quadriceps muscle is more susceptible to aging because it contains more fast-twitch fibers than soleus muscle, therefore, the beneficial effect of MFGM intake with VR may have been more apparent in the quadriceps muscle. The effect of MFGM intake plus VR on the contractile force of quadriceps muscle is still unclear in this study because it was technically unable to measure the contractile force of this muscle in mice with our method. Ota et al. (24) demonstrated that MFGM consumption plus habitual exercise increased the crosssectional area of the quadriceps muscles and improved knee extension strength in human. Considering the previous results, it is possible that MFGM intake with VR improved the contractile force of quadriceps muscle as well as soleus muscle in mice.

In our previous study (29), we showed that continuous intake of MFGM combined with VR diminished ageassociated reductions in motor coordination and muscle strength in 14-mo-old mice, which was associated with increased expression of muscle-derived agrin. The present study revealed that MFGM with VR also improved motor coordination and muscle force in 20- and 21-moold mice, respectively, through increased expression of Dok-7. Dok-7 gene expression in the muscles of 14-mo-old mice was not lower than that of young mice, whereas mRNA and protein expression of Dok-7 in the muscles of 21-mo-old mice was decreased compared to that of young mice. Valdez et al. (45) demonstrated that severe NMJ abnormalities were dramatically increased in the muscles of 18- and 24-mo-old mice. Considering the drastic decrease of time spent on the rotarod, in the present study, 20- to 21-mo-old mice were in the later phase of aging. On the other hand, severe NMJ abnormalities were rarely observed in the muscles of 14-moold mice in our previous study (29); therefore, mice of this age may be during the early phase of aging. In this study, we observed that the age-related reduction in Dok-7 levels in muscle was rescued by MFGM intake with VR in 21-mo-old mice. Considering these findings, the influence of decreased Dok-7 expression may become more apparent as aging progresses, and the effectiveness of increasing levels of Dok-7 via MFGM supplementation may be observed more clearly during the later phase of aging.
MFGM contains membrane-specific proteins and several types of phospholipids, including sphingolipids, any of which may contribute to its effects. We have previously demonstrated that the sphingolipid fractions of MFGM and sphingomyelin increased Dok-7 gene expression in muscle cells (25) and that dietary sphingomyelin intake with moderate exercise improved physical performance in mice (30). These results suggest that sphingomyelin may be the active component that improves the condition of the NMJ, and contributes to the beneficial effects of MFGM. In the present study, exercise increased the efficacy of MFGM intake, consistent with the results of our previous study that used mice during the early phase of aging (29). As discussed in the previous study, sphingolipid metabolism activated by exercise (46) and sphingomyelin metabolites $(47,48)$ may have a significant role in neuromuscular signaling in motor units and muscle regeneration. These findings suggest that MFGM intake and exercise may serve as a sphingolipid source and metabolic activator, respectively. When combined, these factors appear to synergistically improve the efficiency of metabolic turnover, producing the active component of MFGM.

In the present study, 1\% MFGM intake and VR were started when the mice were 2 mo of age and continued for 19 mo. We also observed that $1 \mathrm{~g}$ MFGM consumption and habitual exercise for only $10 \mathrm{wk}$ improved physical performance in human subjects $(22,24)$. The amount of MFGM intake and the intervention period duration required to improve physical performance in humans were much smaller than those that have been examined in mice $(25,29$, and this study). In humans and mice, digestion and absorption rate of sphingolipids, which may be active components of MFGM, are closely equivalent (49); therefore, the minimum-necessary amount of MFGM intake and the minimum-necessary intervention period may be smaller in mice than those using during present study. Also, the quantity and intensity of exercise on the running wheel differed among mice in the present experiment. Consequently, the minimum-necessary exercise quantity and intensity required to realize the beneficial effects of MFGM with exercise remains to be clarified. In order to clarify the points mentioned above, we are presently conducting treadmill experiments which allow us to control the quantity and intensity of exercise that we apply to the mice.

There were still several limitations to the present study, although these do not diminish the importance of our findings. One major limitation is that the effects of MFGM on NMJ morphology in mice during the later phases of aging remain unclear. To address this, we are currently studying the effect of dietary MFGM on the morphological integrity of the NMJ in older mice. Second, the effect of MFGM intake on the brain, as it relates to improved motor coordination, requires further clarification. This is because various brain regions (the motor cortex, basal ganglia, and cerebellum) influence motor coordination (50-52). We are, therefore, presently conducting a detailed examination of the mechanisms 
underlying the beneficial effects of MFGM on motor coordination.

In conclusion, this study provided evidence that continuous supplementation with MFGM combined with VR from a young age ameliorated aging-associated deficits in motor coordination and skeletal muscle function in naturally aging mice in the later phase of aging. This phenomenon may occur via upregulation of Dok7, a molecule involved in maintaining NMJ integrity, in skeletal muscle. Combining physical activity with nutritional intervention, in the form of MFGM, could help to prevent aging-associated declines in motor coordination and muscle function, and thus improve quality of life among older adults.

\section{Disclosure of state of COI}

All authors are employees of the Kao Corporation. This study was supported financially by the Kao Corporation.

\section{Acknowledgments}

We want to thank Hidefumi Kitazawa, Satoko Soga, and Akemi Kobayashi for their excellent technical assistance, and Editage (www.editage.jp) for English language editing.

Michiko Yano and Satoshi Haramizu contributed equally to this work.

This study was supported by Kao Corporation, and no other funding/outside financial support was received.

\section{REFERENCES}

1) Janssen I, Shepard DS, Katzmarzyk PT, Roubenoff R. 2004. The healthcare costs of sarcopenia in the United States. J Am Geriatr Soc 52: 80-85.

2) Breen L, Phillips SM. 2011. Skeletal muscle protein metabolism in the elderly: interventions to counteract the 'anabolic resistance' of ageing. Nutr Metab (Lond) 8: 68.

3) Tieland M, Trouwborst I, Clark BC. 2018. Skeletal muscle performance and ageing. J Cachexia Sarcopenia Muscle 9: 3-19.

4) Seidler RD, Bernard JA, Burutolu TB, Fling BW, Gordon MT, Gwin JT, Kwak Y, Lipps DB. 2010. Motor control and aging: links to age-related brain structural, functional, and biochemical effects. Neurosci Biobehav Rev 34: 721-733.

5) Gonzalez-Freire M, de Cabo R, Studenski SA, Ferrucci L. 2014. The neuromuscular junction: aging at the crossroad between nerves and muscle. Front Aging Neurosci 6: 208.

6) Shigemoto K, Kubo S, Mori S, Yamada S, Akiyoshi T, Miyazaki T. 2010. Muscle weakness and neuromuscular junctions in aging and disease. Geriatr Gerontol Int 10: S137-S147.

7) Jang YC, Lustgarten MS, Liu Y, Muller FL, Bhattacharya A, Liang H, Salmon AB, Brooks SV, Larkin L, Hayworth CR, Richardson A, Van Remmen H. 2010. Increased superoxide in vivo accelerates age-associated muscle atrophy through mitochondrial dysfunction and neuromuscular junction degeneration. FASEB J 24: 1376-1390.

8) Jang YC, Van Remmen H. 2011. Age-associated alterations of the neuromuscular junction. Exp Gerontol 46: 193-198.
9) Campbell MJ, McComas AJ, Petito F. 1973. Physiological changes in ageing muscles. J Neurol Neurosurg Psychiatry 36: 174-182.

10) Morrison S, Newell KM. 2012. Aging, neuromuscular decline, and the change in physiological and behavioral complexity of upper-limb movement dynamics. J Aging Res 2012: 891218.

11) Hillman CH, Erickson KI, Kramer AF. 2008. Be smart, exercise your heart: exercise effects on brain and cognition. Nat Rev Neurosci 9: 58-65.

12) Pedersen BK, Febbraio MA. 2012. Muscles, exercise and obesity: skeletal muscle as a secretory organ. Nat Rev Endocrinol 8: 457-465.

13) Theou O, Stathokostas L, Roland KP, Jakobi JM, Patterson C, Vandervoort AA, Jones GR. 2011. The effectiveness of exercise interventions for the management of frailty: a systematic review. J Aging Res 2011: 569194.

14) Chalé A, Cloutier GJ, Hau C, Phillips EM, Dallal GE, Fielding RA. 2013. Efficacy of whey protein supplementation on resistance exercise-induced changes in lean mass, muscle strength, and physical function in mobilitylimited older adults. J Gerontol A Biol Sci Med Sci 68 : $682-690$.

15) Leenders M, Verdijk LB, van der Hoeven L, van Kranenburg J, Nilwik R, van Loon LJ. 2013. Elderly men and women benefit equally from prolonged resistancetype exercise training. J Gerontol A Biol Sci Med Sci 68 : 769-779.

16) Elliot TA, Cree MG, Sanford AP, Wolfe RR, Tipton KD. 2006. Milk ingestion stimulates net muscle protein synthesis following resistance exercise. Med Sci Sports Exerc 38: 667-674.

17) Wilkinson SB, Tarnopolsky MA, Macdonald MJ, Macdonald JR, Armstrong D, Phillips SM. 2007. Consumption of fluid skim milk promotes greater muscle protein accretion after resistance exercise than does consumption of an isonitrogenous and isoenergetic soy-protein beverage. Am J Clin Nutr 85: 1031-1040.

18) Josse AR, Tang JE, Tarnopolsky MA, Phillips SM. 2010. Body composition and strength changes in women with milk and resistance exercise. Med Sci Sports Exerc 42: 1122-1130.

19) Kukuljan S, Nowson CA, Sanders K, Daly RM. 2009. Effects of resistance exercise and fortified milk on skeletal muscle mass, muscle size, and functional performance in middle-aged and older men: an 18-mo randomized controlled trial. J Appl Physiol 107: 1864-1873.

20) Cavaletto M, Giuffrida MG, Conti A. 2008. Milk fat globule membrane components-a proteomic approach. Adv Exp Med Biol 606: 129-141.

21) Kim H, Suzuki T, Kim M, Kojima N, Ota N, Shimotoyodome A, Hase T, Hosoi E, Yoshida H. 2015. Effects of exercise and milk fat globule membrane (MFGM) supplementation on body composition, physical function, and hematological parameters in community-dwelling frail Japanese women: a randomized double blind, placebocontrolled, follow-up trial. PLoS One 10: e0116256.

22) Minegishi Y, Ota N, Soga S, Shimotoyodome A. 2016. Effects of nutritional supplementation with milk fat globule membrane on physical and muscle function in healthy adults aged 60 and over with semiweekly light exercise: a randomized double-blind, placebo-controlled pilot trial. J Nutr Sci Vitaminol 62: 409-415.

23) Yoshinaka Y, Soga S, Ota N, Yokoyama K, Yamada Y, Kimura M. 2018. Light rhythmic exercise with dietary 
milk fat globule membrane improves physical fitness in an elderly Japanese population: a double-blind randomized placebo-controlled trial. Biosci Biotechnol Biochem 82: 677-682.

24) Ota N, Soga S, Hase T, Shimotoyodome A. 2015. Daily consumption of milk fat globule membrane plus habitual exercise improves physical performance in healthy middle-aged adults. Springerplus 4: 120.

25) Haramizu S, Mori T, Yano M, Ota N, Hashizume K, Otsuka A, Hase T, Shimotoyodome A. 2014. Habitual exercise plus dietary supplementation with milk fat globule membrane improves muscle function deficits via neuromuscular development in senescence-accelerated mice. Springerplus 3: 339.

26) Chiba Y, Shimada A, Kumagai N, Yoshikawa K, Ishii S, Furukawa A, Takei S, Sakura M, Kawamura N, Hosokawa M. 2009. The senescence-accelerated mouse (SAM): a higher oxidative stress and age-dependent degenerative diseases model. Neurochem Res 34: 679-687.

27) Drechslers S, Lynch MA, Novella S, González-Navarro H, Hecimovic S, Barini E, Tucci V, Castro RE, Vandenbroucke RE, Osuchowski M, Potter PK. 2016. With mouse age comes wisdom: A review and suggestions of relevant mouse models for age-related conditions. Mech Ageing Dev 160: 54-68.

28) Justice JN, Carter CS, Beck HJ, Gioscia-Ryan RA, McQueen M, Enoka RM, Seals DR. 2014. Battery of behavioral tests in mice that models age-associated changes in human motor function. Age (Dordr) 36: 583-592.

29) Yano M, Minegishi Y, Sugita S, Ota N. 2017. Milk fat globule membrane supplementation with voluntary running exercise attenuates age-related motor dysfunction by suppressing neuromuscular junction abnormalities in mice. Exp Gerontol 97: 29-37.

30) Haramizu S, Ota N, Otsuka A, Hashizume K, Sugita S, Hase T, Murase T, Shimotoyodome A. 2014. Dietary milk fat globule membrane improves endurance capacity in mice. Am J Physiol Reg Integr Comp Physiol 307: R1009-R1017.

31) Fernandez AM, de la Vega AG, Torres-Aleman I. 1998. Insulin-like growth factor I restores motor coordination in a rat model of cerebellar ataxia. Proc Natl Acad Sci USA 95: 1253-1258.

32) Haramizu S, Ota N, Hase T, Murase T. 2011. Catechins attenuate eccentric exercise-induced inflammation and loss of force production in muscle in senescence-accelerated mice. J Appl Physiol 111: 1654-1663.

33) Plotnik M, Giladi N, Hausdorff JM. 2007. A new measure for quantifying the bilateral coordination of human gait: effects of aging and Parkinson's disease. Exp Brain Res 181: 561-570.

34) Soga S, Ota N, Shimotoyodome A. 2015. Dietary milk fat globule membrane supplementation combined with regular exercise improves skeletal muscle strength in healthy adults: a randomized doubleblind, placebo-controlled, crossover trial. Nutr J 14: 85.

35) Ishihara A, Roy RR, Ohira Y, Ibata Y, Edgerton VR. 1998. Hypertrophy of rat plantaris muscle fibers after voluntary running with increasing loads. J Appl Physiol (1985) 84: 2183-2189.

36) Gallo M, Gordon T, Syrotuik D, Shu Y, Tyreman N, MacLean I, Kenwell Z, Putman CT. 2006. Effects of long-term creatine feeding and running on isometric functional measures and myosin heavy chain content of rat skeletal muscles. Pflugers Arch 452: 744-755.

37) Legerlotz K, Elliott B, Guillemin B, Smith HK. 2008. Voluntary resistance running wheel activity pattern and skeletal muscle growth in rats. Exp Physiol 93: 754-762.

38) Arimura S, Okada T, Tezuka T, Chiyo T, Kasahara Y, Yoshimura T, Motomura M, Yoshida N, Beeson D, Takeda S, Yamanashi Y. 2014. Neuromuscular disease. DOK7 gene therapy benefits mouse models of diseases characterized by defects in the neuromuscular junction. Science 345: 1505-1508.

39) Okada K, Inoue A, Okada M, Murata Y, Kakuta S, Jigami T, Kubo S, Shiraishi H, Eguchi K, Motomura M, Akiyama T, Iwakura Y, Higuchi O, Yamanashi Y. 2006. The muscle protein Dok-7 is essential for neuromuscular synaptogenesis. Science 312: 1802-1805.

40) Inoue A, Setoguchi K, Matsubara Y, Okada K, Sato N, Iwakura Y, Higuchi O, Yamanashi Y. 2009. Dok-7 activates the muscle receptor kinase MuSK and shapes synapse formation. Sci Signal 2: ra7.

41) Yamanashi Y, Higuchi O, Beeson D. 2008. Dok-7/MuSK signaling and a congenital myasthenic syndrome. Acta Myologica 27: 25-29.

42) Garcia N, Santafe MM, Tomas M, Priego M, Obuis T, Lanuza MA, Besalduch N, Tomas J. 2012. Exogenous ciliary neurotrophic factor (CNTF) reduces synaptic depression during repetitive stimulation. J Peripher Nerv Syst 17: 312-323.

43) Chai RJ, Vukovic J, Dunlop S, Grounds MD, Shavlakadze T. 2011. Striking denervation of neuromuscular junctions without lumbar motoneuron loss in geriatric mouse muscle. PLoS One 6: e28090.

44) Thompson LV. 2009. Age-related muscle dysfunction. Exp Gerontol 44: 106-111.

45) Valdez G, Tapia JC, Kang H, Clemenson GD Jr, Gage FH, Lichtman JW, Sanes JR. 2010. Attenuation of agerelated changes in mouse neuromuscular synapses by caloric restriction and exercise. Proc Natl Acad Sci USA 107: 14863-14868.

46) Błachnio-Zabielska A, Baranowski M, Zabielski P, Górski J. 2008. Effect of exercise duration on the key pathways of ceramide metabolism in rat skeletal muscles. J Cell Biochem 105: 776-784.

47) Brailoiu E, Cooper RL, Dun NJ. 2002. Sphingosine 1-phosphate enhances spontaneous transmitter release at the frog neuromuscular junction. Br J Pharmacol 136: 1093-1097.

48) Danieli-Betto D, Germinario E, Esposito A, Megighian A, Midrio M, Ravara B, Damiani E, Libera LD, Sabbadini RA, Betto R. 2005. Sphingosine 1-phosphate protects mouse extensor digitorum longus skeletal muscle during fatigue. Am J Physiol Cell Physiol 288: C1367-C1373.

49) El-Loly MM. 2011. Composition, properties and nutritional aspects of milk fat globule membrane. Pol J Food Nutr Sci 61: 7-32.

50) Houk JC, Wise SP. 1995. Distributed modular architectures linking basal ganglia, cerebellum, and cerebral cortex: their role in planning and controlling action. Cereb Cortex 5: 95-110.

51) Middleton FA, Strick PL. 2000. Basal ganglia and cerebellar loops: motor and cognitive circuits. Brain Res Brain Res Rev 31: 236-250.

52) Tyč F, Boyadjian A. 2011. Plasticity of motor cortex induced by coordination and training. Clin Neurophysiol 122: 153-162. 